\title{
XXXI. The photo-electric theory of vision
}

\section{J.H.J. Poole M.A.}

To cite this article: J.H.J. Poole M.A. (1921) XXXI. The photo-electric theory of vision, Philosophical Magazine Series 6, 41:243, 347-357, DOI: 10.1080/14786442108636227

To link to this article: http://dx.doi.org/10.1080/14786442108636227

曲 Published online: 08 Apr 2009.

Submit your article to this journal $\pi$

Џll Article views: 3

Q View related articles $\square$

Citing articles: 2 View citing articles 5 
Obliquely Incident Light.-When the film is tilted relatively to the clirection of the incident rays, the rings seen on the film contract and move inwards. As in the case of normal incidence, perfectly black rings may be seen on the film, if it be viewed in monochromatic light very nearly in the direction of the transmitted rays, and the appearance of these rings alters with the obliquity of observation in much the same way. It should be remarked, however, that in the present case the effects observed vary not only with the angle between the transmitted pencil and the direction of observation, but also with the particular plane in which the latter direction lies. The maximum permissible angle of observation varies with this plane. In the plane of incidence it is $\pi / 2-\alpha$ and $\pi / 2+\alpha$ respectively on the two sides of the transmitted pencil, where $\alpha$ is the ang'e of incidence. If $\alpha$ is considerable, the rings continue to be visible when the film is viewed nearly along the surface of the plate on one side of the transmitted pencil, while on the other side the rings move out and disappear from the film at a moderate obliquily, so that in white light the film appears achromatic over a wide range of angles of observation.

Calcutta, India, 18th September, 1920.

XXXI. The Photo-Electric Theory of Vision.
By J. H. J. PooLe, M.A.*

$\coprod \mathrm{N}$ $\mathrm{N}$ his address to the British Association in 1919, Sir Oliver Lodge suggested that the light sensation in the eye might be caused by the action of photo-electrons excited by the incident light. He thought that, if experiments were carried out on the various substances present in the retina, some of them might be found to exhibit photoelectric properties when exposed to visual light. The same idea had occured to Dr. Joly some years previously, and in 19.15-16 some experiments were actually carried out in this laboratory on the possible photo-electric properties of the biack pigment No evilence of the black pigment possessing any photo-electric powers, when exposed to visual light, could however be obtained, and as thus no direct experimental evidence in favour of the theory could be given, Dr. Joly refrained from publishing this view of the mechanism of

* Comnunicated by Prof. J. Joly, F.R.S. 
vision. When, however, Sir Oliver Lodge independently proposed the same theory, it was thought worth while repeating the experiments more fully, and seeing if any definite eridence in favour of the theory could be obtained.

It seems highly probable, it might almost be said certain, that the effect of light on the eye must ultimately be due to a photo-electric action of some kind ; but the real foint of issue is whether it can be shown that the photo-electron excited by the light ever gets free of its parent molecule as it does when a metal is illuminated with ul ra-violet light, or whether the action is less violent and may only result in disturbing the equilibrium of the electron inside the molecule, and hence altering the chemical properties of some light-sensitive compound in the eye. The latter view would favour such a theory of vision as the Young-Helmholtz theory, which postulates the existence of three definite and independent colour-sensations in the eye, which might be caused by three different light-sensitive compounds; but if it could be shown that the former view were correct, a very much more simple and direct explanation of colour-vision can be given, at least from the physical point of view. This explanation rests on the fact that it has been shown that the velocity of emission of a photo-electron is a simple function of the wavelength of the incident light-in fact, that the energy of the electron is given by the equation $\mathrm{E}=h \nu-p$, where $h$ is Planck's constant, $\nu$ is the frequency of the light, and $p$ is the work done in getting the electron free from the molecule and will thus be a constant for any particular material. Thus for every different colour in the spectrum an electron of a definite velocity would be emitted, and it is only necessary to suppose that the manner in which the cones are excited depends on the velocity of these electrons, to obtain a possible view of the mechanism of colour-vision. It might, perhaps be urged against such a theory as this, that on this view the eye ought always to be able to distinguish between a pure single frequency light and an impure mixture of two different frequency lights, which, as is well known, the eye is in certain cases incapable of doing: $e ., g$, it cannot distinguish between spectrum yellow and a suitable mixture of green and red. However, we could meet this objection by supposing that the resolving power of the cones for photoelectrons of various velocities was not perfect, and that they were only capable of separating the electrons into three main groups, which would correspond to the three primary sensations of red, green, and blue. Thus in the case of spectrum yellow, which lies between red and green, the resultant 
electrons would excite both the red and green sensations and hence such a colour would be visually indistinguishable from a mixture of red and green. In fact, if we adupt this view of the resolving power of the cones for photo-electrons of different velocities, we could explain all the facts which agree with the Yomg-Helmboltz theory of three primary colour sensations.

Before maling any direct tests of the possible plotoelectric powers of the retina, some prelininary indirect trials were made. It seems probable that the formation of the latent image in a photographic plate is really based on a photo-electric action due to the silver halides which are found to be vigorously photo-electric. Sensitisers are also photoelectric, their activity being dependent on the absorption of light of some particular colour. It is, therefore, perhaps permissible to infer that if either the black pignent or rhodopsin were really photo-electric to visual light, they would also be capable of acting as sensitisers for a photographic plate, it treated in a suitable manner. Experiments on this point were accordingly made by exposing a plate which was insensitive to the red end of the spectrum to intense red light. One half of the plate was treated with the preparation from the eye, which it was desired to test. The untreated portion acted as a standard for comparison purposes, and by putting a slight scratch with a penknife on the treated half it could always be subsequently identified. An ordinary carbon glow-lamp was used as a source of light. Before falling on the plate, the light passed through a picric-acid screen, ordinary photographic ruby glass, and also a special red screen, as it was found that the ruby glass allowed quite a large proportion of the blue end of the spectrum to pass through.

The first material to be dealt with was the black pigment. Varions methods of applying it to the plate were tried, but in no case was detinite positive evidence of any sensitizing action obtained. In the first attempts the black pigment was simply removed from a freshly dissected bull's eye, and placed directly in contact with the sensitive film. The plate was exposed through the glass, as owing to the opacity of the black pigment no effect could be expected if the black pigment coating was between the film and the incident light. After exposure, the pigment was carefully washed off and the plate given a thorough rinsing with water to remove as far as possible any trace of grease. As already stated however, no sign of any sensitising action could be discovered on development, and in fact 
sometimes the reverse effect was observed, when a sufficiently long exposure to slightly fog the plate was tried. This slight fogging of the plate with long exposures was probably due to a very small amount of actinic liglit getting through the screens, and the retarding action of the pigment observed may be caused by its slighitly greasy nature impeding development. Other attempts were also mad $\theta$ by treating the pigment with various solvents, such as water, alcohol, chloroform, etc., and treating a plate with the resuitant fine suspension of the black pigment, as it did not appear to be soluble in any of the solvents used. No result wis obtained. Another method adopted was to reduce the pigment to a very fine powder $l, y$ dehydration and subsequent grinding in an :gate mortar, the resulting powder being then dusted on to the plate; but again no ser sitising effect was shown. The difficulty of obtaining good enough contact between the pigment and the sensitive film of the plate would probably be sufficient to account for the negative result in all these cases, as it has been found that for ordinary sensitising iy es to be effective, the dye must actually dye the silver-halide grain, and not merely strin the plate.

It is more difficult to deal with the visual purple or rhodopsin in the retina, both on account of the much smaller quantity available, and also because the rhodopsin is only present in dark-adapted eyes. For this reason the animal from which the eye is to be obtained must be kept in the dark for some time before being killed, to allow the rhodopsin to form in the retina, and all subsequent dissections have to be carried out in the dark or in a very subdued red light, as the rhodopsin quickly fades when exposed to light. Proftssor Pringle was, however, kind enough to supply us with a solution of rhodopsin in bile salts which had been prepared from dark-adapted irogs' eyes. This solution was tested on the plate in the usual manner. Again no sensitising action was detected : in fact, with very long exposures which slightly fogged the plate, a small retarding effect was noticed. This effect was found to be due to the bile salts, - a solution of them with no rhodopsin having the same slightly retarding effect on the plate. It is unfortunate that bile sults have this action on the plate, as it would completely mask any slight sensitising effect which the very small amount of rhodopsin present might produce, and it is apparently not easy to extract the rhodopsin from the retina by other means.

These sensitising experiments thus lead to no direct 
evidence in favour of the photo-electric theory of vision, but, on the other hand, it certainly would not be justifiable to conclude from them that the materials tested have no photo-electric properties, since the difficulties of getting intimate contact with the sensitive film are, as already mentioned, probably quite sufficient to account for the nul results obtained.

After the failure of these experiments to show any positive effects, a direct method of testing the black pigment etc. was used.

The usual plan of doing this was employed. The material to be tested is placed on an insulated conducting plate, and the rate of leak of negative electricity from the plate when it is illuminated measured with an electrometer. To facilitate

Fig. 1.

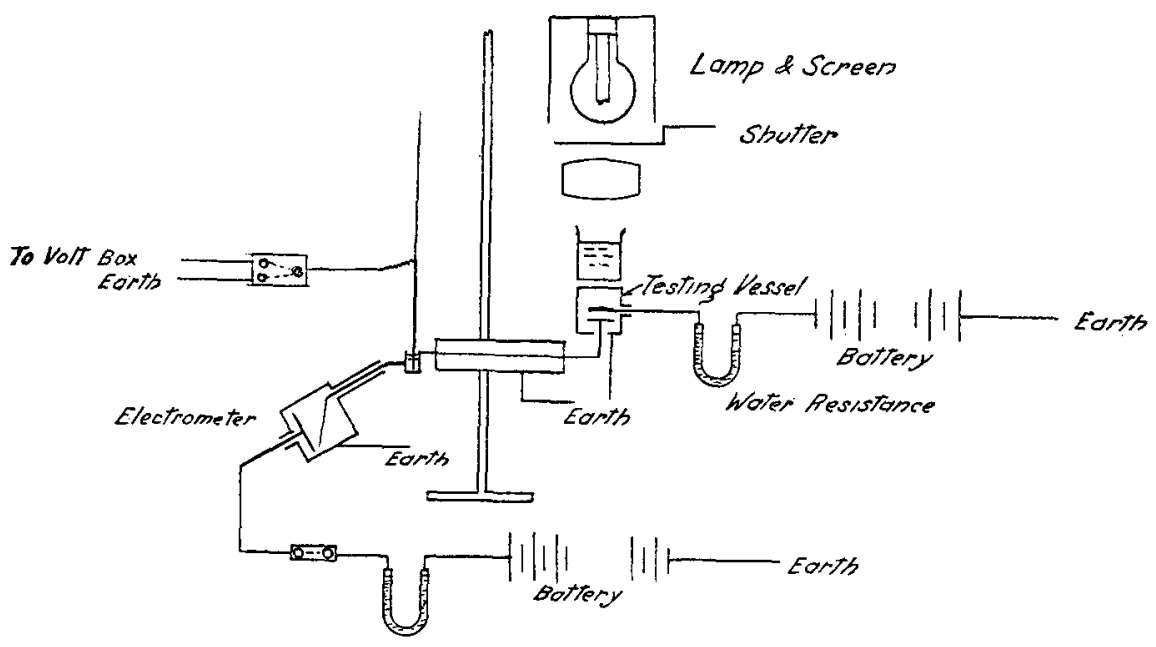

the escape of the negative electrons, an accelerating electric field is generally used. A sketch of the various electrical connexions is given. The Wilson Kaye Tilted Leaf Electrometer was used to ineasure the electronic current. Its high sensibility to voltage changes and small capacity render it peculiarly suitable for this purpose. Fortunately it was found unnecessary to work with the instrument in its most sensitive condition, as the natural leak in the ionizationchamber limits the sensitivity at which it is desirable to work. Usually it was found that a sensitivity of about 30 or 40 scale-divisions to the volt was sufficient, though 
on occasion sensitivities up to 130 divisions per volt were used. To maintain the charged plate of the electrometer at the requisite voltage, a battery of small Ever-Ready dry cells was employed. This battery wa; carefully insulated from earth, the individual cells being stood en a layer of paraffinwax, and its voltage was found to remain constant over long periods so long as no current was taken from it. To protect it in case of an accidental short-circuit due to the gold-leaf touching the charged plate, a water-resistance is inserted between it and the latter. A switch is also inserted for convenience in working with the instrument.

For earthing the gold-leaf when requisite, a copper rod dipping into a strong $\mathrm{CaCl} \mathrm{l}_{2}$ solution contained in the small copper cup supplied with the instrument was used. When it was desired to isolate the gold-leaf, the copper rod could be lifted by a silk thread passing over a suitable arrangement of pulley-wheels. On the whole this key proved fairly satisfactory, but at times it was inclined to give too big an initial charge to the gold-leaf. Usually, however, after two or three attempts, a small enough displacement could be obtained.

In order to conveniently measure the sensitivity of the electrometer, the copper rod of the earthing key was not directly connected to earth, but was connected to a two-way switch so that a known voltage could be applied to it by means of a volt-box. Thus the sensitivity of the electrometer can be quickly determined at any time, which is essential as any small accidental shake of the instrument may alter it considerably, and for this reason it is necessary to test it fairly frequently.

A separate sketch of the final form of testing-chamber is shown. The chamber was constructed of brass, and made in two parts so that it could bo easily taken apart to insert the material under test. The joint was carefully ground with carborundum-powder and tallowed, in order to insure the vessel being air-tight when assembled. It could be exhausted through a side tubulure, which was connecied to a Geryk pump and manometer. Pressures down to about $3 \mathrm{~mm}$. of mercury could be maintained in the testingchainber.

The black pigment or other material is piaced on the small brass testing-plate. This plate is carried on a copper rod which projects slightly from the bottom of the vessel and is connected to the electrometer by a fine wire. The wire is surrounded by an earthed shield to prevent any stray electrostatic effects due to the observer etc. affecting 
the electrometer. The copper rod itself is insulated from the main body of the chamber, which is earthed, by sealingwax and a quartz tube as depicted. The sealing-wax serves to make the joint air-tight. Abont $3 \mathrm{~mm}$. above the testingplate, a small quartz plate carried by a brass ring is fitted. The latter is supported by a small steel rod, which passes through the side of the vessel and is also insulated with a glass tube and sealing-wax. This plate replaces the ordinary grid of copper gauze or other form of grating usually omployed to collect the negative ions escaping from the phuto-electric surface. By arplying a thin film of a fairly strong solution of either $\mathrm{H}_{2} \mathrm{SO}_{4}$ or $\mathrm{P}_{2} \mathrm{O}_{5}$ to the lower surface of the plate, it can be made sufficiently conducting for working with the high voliages and small currents met with in these experiments. The outer brass ring which supports.

Fig. 2.

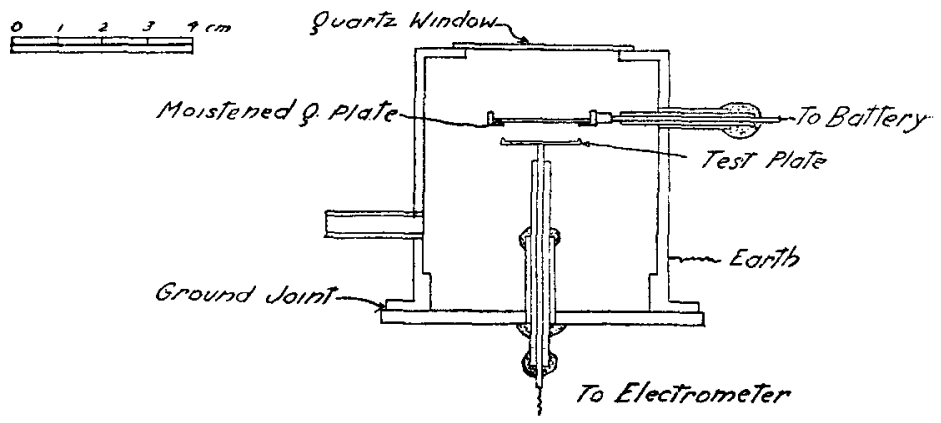

the plate also serves to make contact with the liquid film. To facilitate the escape of the electrons from the test plate the upper quartz plate is connected through its steel supporting rod to the positive pole of a high-voltage battery. At low pressures the potential gradient was high enough to largely increase the electronic current by ionization due to collision, but in experiments carried ont at air-pressure this would not be the case.

The use of the moistened quartz plate, instead of the usual form of wire grid, has some great advantages. The most obvious one lies in its superior transparency, but it also possesses a great virtue in that its shape is much more constant than that of the wire grid. The importance of this fact has been pointed out by H. H. Dixon and H. H. Poole in a paper on "Photo-Synthesis and the Electronic Theory" (Proc. Roy. Dublin Soc. vol. xvi. N. S. No. 5), in which they show that the change in capacity due to the sagging of a 
wire-gauze grid when heated by the incident light may produce apparently quite large photo-electric effects, which are really quite spurious. 'The quartz plate, however, will manifestly not suffer from this complaint on at least three accounts. These are: (a) The thermal expansion of quartz is practically zero; (b) Owing to its transparency it will absorb very little energy from the incident beam; and (c) Even if the plate did expand slightly it would do so in its own plane and not alter the capacity of the isolated system appreciably. The chief drawback of the arrangement is that it cannot obviously be used at very low pressures owing to the evaporation of the liquid film.

The test-plate was illuminated through a quartz window at the top of the testing vessel. A quartz window was employed, and it is convenient to be able to use ultraviolet light on occasion to test whether the arrangement is funcioning properly. As a source of light a 200-watt $\frac{1}{2}$-watt lamp was employed, and a suitable train of lenses used to concentrate the light. A cardboard screen was placed round the lamp to protect the observer's eyes, and the electrometer was completely shielded from the light by a large wooden screen. It was found that switching on this light had a slight effect on the electrometer, although the leads to the lamp were kept as far distant as possible. In consequence, a shutter was used by which the illumination could be controlled without turning the lamp off.

In order to obtain some idea of the absolute current to which a known rate of deflexion of the gold-leaf corresponds, it is necessary to measure the capacity of the gold-leaf and testing plate taken in conjunction. This capacity was determined by comparing it with a second capacity whose value cuuld be calculated approximately. The method adopted was that described by H. H. Dixon and H. H. Poole in their paper on Photo-Synthesis (loc. cit.). The capacity was found to be about $6 \mathrm{cms}$., and as the sensitivity is also known we can easily determine the value of the currents obtained. The value obtained for the capacity is probably not very accurate, but great accuracy is not requisite, as really all we require to know in these experiments is the order of magnitude of the currents dealt with.

'The method of procedure adopted was first to spread a thin film of the substance to be tried on a disk of pure leadtoil, which was then placed on the testing plate of the ionization chamber. The chamber was re-assembled, and the pump worked till the desired pressure had been attained. The rate of leak of the electrometer with the light off and on 
was then measured in the usual way. The advantage of using lead disks to place the active material on is that it makes the manipulation of the latter easier, and also, when one preparation has been tested, the disk can be quickly removed from the testing vessei and a second one substituted. Lead is a convenient metal to use, as it is easily cut to the required shape, and is also very inert photoelectrically.

The experiments carried out on the black pigment with this apparat is completely confirmed the nul results previously obtained with it. Various methods of preparing the pigment were tried. It was tested moist and after being dried in a desiccator, immediately after dissection and after the lapse of some time, but in no cuse was any photo-electric effect obtained. A test was also made on a complete retina, which was spread entire on one of the lead disks, but it also showed no photo-electric powers. All these experiments were conducted at a pressure of about $5 \mathrm{~mm}$. of mercury. There can, it seems, be very little doubt that the black pigment of the eye is not photo-electric when exposed to visual light.

As regards the rhodopsin in the retina, it was thought best to test it in situ in the retina. This was done by testing a freshly prepared retina obtained from a frog's eye. The frog, before the experiment, had been kept in the dark, so that there was a certain amount of rhodopsin present in the retina. Professor Pringle kindly did the requisite dissections which were carried out in feeble red light, the retina not being exposed to ordinary illumination at all till it was tested. As it was considered that perhaps the vacuum in the testing vessel might tend to render the retina inactive owing to its drying action, these trials were all carried out at air pressure.

The result of these experiments showed that while the frog's retina certainly possesses no permanent photo-electric effect, there is usually a very small effect for a few minutes after the retina is first placed in the testing vessel. In all cases such an effect was found, but its size was very small and irregular, varying from about $6 \times 10^{-6}$ E.S.U. to $0.5 \times 10^{-6}$ E.S.U.

In view of the small size of the currents obtained, it is perhaps of interest to consider what wonld be the smallest number of electrons per second which the human eye could be expected to detect. If we assume that a candle is visible at night at a distance of 3000 metres, and that the effective aperture of the eye in such a case would be about $1 \mathrm{sq} . \mathrm{cm}$., we find that the minimum visible energy-flow is equal to 
about $4 \times 10^{-7}$ erg per second, taking the energy emitted by the unit candle as $5 \times 10^{5}$ eros per second. Let us also assume that the average wave-length is about $5000 \mathrm{~A} . \mathrm{U}$. (this is probably too short, but is sufficiently accurate for thís purpose), and we find that the energy of the corresponding photo-e'ectron on the quantum theory will be equal to $h \nu=3 \cdot 9 \times 10^{-12} \mathrm{erg}$.

If all the energy of the incident light is converted into energy of the photo-electrons, this would lead to about $10^{5}$ electrons per second being the minimum number visible, and a current of $5 \times 10^{-5}$ E.S.U. being the minimum electronic current.

In comparing this latter figure with the actual small current obtained from the frog's retina, there are several considerations to be taken into account: i.e., (a) a frog's eye is very much smaller than a human eye; (b) the illumination was not the minimum, but was intensely bright. Also, in any case, we could not expect to get anything approiching the theoretically possible current owing to absorption of the electrons in the surface, loss of energy by reflexion, and also the fact that even under ideal conditions the total incident energy may not be entirely converted into that of the emitted electrons. As regards $(a)$ and $(b)$, the actual energy falling on the frog's retina per second was approximately $10^{1}$ ergs per second, or $2.5 \times 10^{10}$ times that required to excite the human eye. This would give a theoretically possible current of $1.25 \times 10^{6}$ E.S.U., or about $2 \times 10^{11}$ times the actual current obtained. It is nossible that absorption in the surface might account for the enormous discrepancy between these two figures, but certainly no other consideration could do so. In this connexion some results obtained by Elster and Geitel (Phys. Zeits. xiii. p. 468, 1912) are of interest. By the use of a very sensitive potassium cell, they were able to detect an amount of blue light imparting $3 \times 10^{-7} \mathrm{erg}$ per second per sq. cm., and they found that under these conditions the energy of the emitted electrons accounted for $\frac{1}{30 \sigma}$ part of the incident energy. As it has been found that the photo-electric current for a substance is usually a linear function of the light intensity, it would from this appear probable that in all cases of photo-electric action a sensible proportion of the incident energy would be represented in the energy of the emitted electrons. Thus we are driven to conclude that since in the case of the retina only $5 \times 10^{-12}$ part is so represented, it cannot be considered to be photo-electric. The current obtained is 
Active Leposits of Radium, Thorium, and Actinium. 357

so small that it may possibly be due to some extraneous effect due to heating etc.

From these experiments we may therefore safely conclude that neither the black pigment nor the retina as a whole is photo-electric to visual light. It is possible however that, as previously mentioned, the nul effects obtained with the retina may be due to absorption of the electrons in the surface. It remains quite conceivable that the rhodopsin in the eye is actually photo-electric, but so immersed in inactive material that the electrons cannot escape. Thns the photo-electric theory of vision, while still presenting many attractions, cannot be said to be in any way confirmed by these experiments, but neither can it be actually refuted by them. To obtain really conclusive evidence it would be necessary to isolate sufficient rhodopsin in a pure state to be able to test it directly.

In conclusion I wish to express my thanks to Professor Pringle and Dr. Fearon for their kind aid in the preparation of the frogs' retinas; also to Dr. Joly, to whose suggestion and assistance the research is mainly due.

Iveagh' Geological Laboratory,

November 1920.

XXXII. The Nistribution of the Active Deposits of Radium, Thorium, and Actinium in Electric Fields. By G. H. BrIgGs, B.Sc., Lecturer in Physics at the University of Sydney*.

\section{Introduction.}

1) UE experiments to be described in this paper were 1 begun with the object of deciding whether, as E. M. Wellish $\nmid$ had concluded, there is a definite limiting fraction of the recoil atoms from radium emanation, positively charged at the end of the recoil path, or whether, as G. H. Henderson concluded, all the recoil atoms from thorium and radium emanations are positively charged at the end of the recoil path. As the work progressed, it was found necessary to extend its scope.

In his experiments Wellish used a cylindrical vessel with a central electrode. He obtained the following results for the recoil atoms of radium emanation : 88.2 per cent.

* Communicated by the Author.

$\dagger$ Wellish, Phil. Mag. xxviii. p. 417 (1914).

Phil. Mag. S. 6. Vol 41. No. 243. March 1921. 